\title{
Research Article \\ Stability Analysis Based on Caputo-Type Fractional-Order Quantum Neural Networks
}

\author{
Yumin Dong $\mathbb{D}$, Xiang Li $\mathbb{D}^{\text {, }}$, Wei Liao $\mathbb{D}$, and Dong Hou \\ College of Computer and Information Science, Chongqing Normal University, Chongqing 401331, China \\ Correspondence should be addressed to Yumin Dong; dym@cqnu.edu.cn
}

Received 12 April 2021; Revised 17 May 2021; Accepted 2 June 2021; Published 28 June 2021

Academic Editor: Chun Lu

Copyright (C) 2021 Yumin Dong et al. This is an open access article distributed under the Creative Commons Attribution License, which permits unrestricted use, distribution, and reproduction in any medium, provided the original work is properly cited.

\begin{abstract}
In this paper, a quantum neural network with multilayer activation function is proposed by using multilayer Sigmoid function superposition and learning algorithm to adjust quantum interval. On this basis, the quasiuniform stability of fractional quantum neural networks with mixed delays is studied. According to the order of two different cases, the conditions of quasi uniform stability of networks are given by using the techniques of linear matrix inequality analysis, and the sufficiency of the conditions is proved. Finally, the feasibility of the conclusion is verified by experiments.
\end{abstract}

\section{Introduction}

Fractional calculus is an arbitrary extension of integer calculus in order. It has strong advantages and wide application prospects in the fields of physics, chemistry, biology, economy, control, signal, and image processing. It has attracted extensive attention from scholars at home and abroad and has become one of the current research hotspots. In recent years, due to the continuous development of fractional differential equations, many researchers began to pay attention to the fractional-order theory, and the combination of fractional-order and neural network give full play to the advantages of fractional order. For example, literature [1-4] combined fractional order with neural network and achieved a good effect. Among them, Boroomand and Menhaj [4] presented the fractional-order Hopfield neural network model and studied its stability through the quasienergy function. [5-7] study on different fractional-order neural networks and explore the influence of different factors on fractionalorder neural networks. This paper summarizes the synchronization problem of neural network [8-12]. Dominik et al. [13] considered discrete fractional-order artificial neural networks. Chaos and chaotic synchronization of fractionalorder neural networks are proposed [14]. Literature [15, 16] explained and analyzed the dynamics of fractional-order neural networks. The fractional-order neural network was applied in different fields [17-21]. In recent years, the stability of fractional-order neural network system has become a research hotspot [22-31]. In reference [22], the stability and passivity of a memristor-based fractional-order competitive neural network (MBFOCNN) are analyzed by using Caputo's fractional derivative. The effectiveness of the proposed results is finally verified by using analysis techniques and other computational tools. In reference [23], the problem of robust dissipation of Hopfield-type complex valued neural network (HTCVNN) model with time-varying delay and linear fractional uncertainty is studied, and many numerical models are designed to verify the results. In reference [24, 25], the global asymptotic stability of fractional quaternion numerical bidirectional associative memory neural networks (FQVBAMNNs) and fractional quaternion numerical memristic neural networks (FOQVMNNs) is studied. The effectiveness of the results is proved by using related methods. In reference [26, 27], the stability of fractional-order continuous time quaternion numerical leaky integral echo state neural network (NN) with multiple time-varying delays is studied, and the feasibility of the method is verified by numerical examples. In reference [28], the uniform stability of a fractional-order leaky integral echo state neural network (FOESN) with multiple delays is studied. The simulation results show the effectiveness of the method. Literature [32, 33] proposed the time-delay correlation study of Caputo 


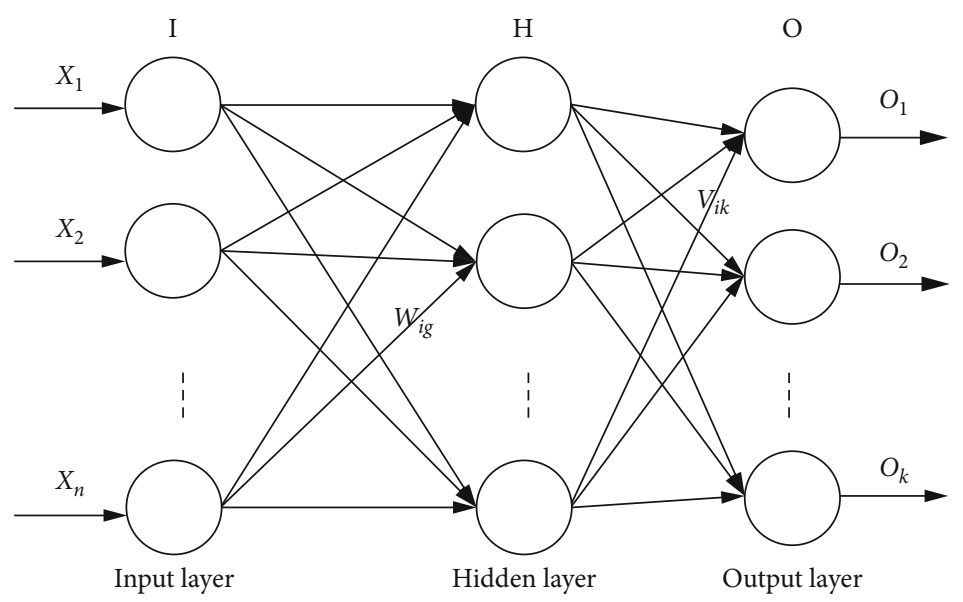

Figure 1: Three-layer feedforward neural network graph structure.

fractional-order neural network. However, there are few studies on the behavior of fractional quantum neural networks with mixed delay. In this paper, a multilayer activation function quantum neural network model is presented, and the quasiuniform stability of fractional quantum neural networks with mixed delay is studied. It is proved by the formula and simulated by the numerical case.

This article is organized as follows. In the second section, we give the structure of the multilayer activation function of the quantum neural network, based on which a fractional quantum neural network model with mixed delay is proposed. In the third section, it is proved that the fractional quantum neural network system with mixed time delay is quasiuniformly stable by corresponding definitions and lemmas. In the fourth section, a concrete example is given to verify the validity and applicability of the given results.

\section{Model Composition and Preparation}

2.1. Quantum Neural Network. Quantum neural network belongs to the feed-forward type of neural network [34, 35]. Compared with the traditional feed-forward type of neural network, the neurons in the hidden layer of quantum neural network refer to the idea of quantum state superposition in the quantum theory and carry out the linear superposition of several Sigmoid functions, which is called the multilayer activation function. Traditional activation functions can only represent two states and orders of magnitude. When quantized, a hidden layer neuron can represent more states and orders of magnitude.

Each Sigmoid function superimposed has a different quantum interval. By adjusting the quantum interval, the data of different classes can be mapped to different orders of magnitude or steps, so that the classification can have more degrees of freedom. The quantum interval of the quantum neural network can be obtained by training. The uncertainty in the sampled data can be obtained and quantified by a quantum neural network with an appropriate learning algorithm.

Figure 1 shows a traditional three-layer feedforward neural network. Assume that the input layer I has $n$ nodes, the output layer $\mathrm{O}$ has $k$ nodes, and the number of nodes in the hidden layer $\mathrm{H}$ is $m$. Adjacent layer nodes are fully interconnected, and nodes of the same layer are not connected. The node output function in the hidden layer is

$$
H_{r}=f\left(\mathbf{W}^{\mathrm{T}} \mathbf{X}-\theta\right) r=1,2, \cdots, u .
$$

The output function of the node in the output layer is

$$
O_{i}=f\left(\mathbf{V}^{\mathrm{T}} \mathbf{H}-\mathrm{h}\right) j=1,2, \cdots, n .
$$

In the formula, $f$ adopts Sigmoid function, and $W$ is the connection weight vector between each neuron in the input layer and each neuron in the hidden layer. $\mathbf{V}$ is the connection weight vector between each neuron in the hidden layer and each neuron in the output layer; $\theta$ is the threshold of the hidden layer, and $h$ is the threshold of the unit of the output layer.

Quantum neural networks with multiple excitation functions:

$$
H r=\frac{1}{n} \sum_{s=1}^{n} f\left[\mathrm{U}\left(\mathbf{W}^{\mathrm{T}} \mathbf{X}-\theta_{s}\right)\right] .
$$

In the formula: $f(x)=1 /(1+\exp (-x)), \mathbf{W}$ is the network weight vector; $\mathbf{X}$ is the network input vector; $U$ is the slope; $\mathbf{W}^{\mathrm{T}} \mathbf{X}$ is the input excitation of the quantum neuron; $\theta_{s}$ is the quantum interval $(s=1,2, \cdots, n)$.

The learning of quantum neural network can be divided into two steps: (1) adjusting the weight to make the input data correspond to different class spaces; (2) adjust the quantum interval of quantum neurons in the hidden layer to reflect the uncertainty of data. The BP algorithm is used to adjust the weight. Once the network weight is obtained, the quantum interval can be adjusted by an appropriate algorithm [36]. The idea of the algorithm is to minimize the output change of the hidden layer neurons in the quantum neural network based on the same kind of sample data. 
Assume that for class $C_{m}$, the output of the $i$ th hidden layer neuron changes as:

$$
e_{i, m}^{2}=\sum_{x_{k}} \sum_{x_{k} \in C_{m}}\left(\left\langle O_{i, m}\right\rangle-O_{i, k}\right)^{2}
$$

in the formula: $O_{i}, k$ represents the output of the $i_{\text {th }}$ neuron in the hidden layer when the network input vector is $x_{k}$;

$$
\left\langle O_{i, m}\right\rangle=\frac{1}{\left|C_{m}\right|} \sum_{x_{i} x_{k} \in C_{m}} O_{i, k} .
$$

$|\mathrm{Cm}|$ in the formula represents the cardinality of class $|\mathrm{Cm}|$. It can be seen that $\mathrm{e}_{i, m}^{2}$ is a function of the quantum interval $\theta_{s}$. By taking the derivative of $\theta_{s}(s=1, \cdots, n)$ on both sides of Equation (4) and finding the minimum value of $\mathrm{e}_{i, m}^{2}$, the variation formula of $\theta_{i, s}$ (i.e., layer $\mathrm{S}$ of the $i$ th neuron in the hidden layer) can be obtained.

$$
\begin{gathered}
\Delta \theta_{i, s}=Z \frac{U}{n S} \sum_{m=1 x_{k}}^{k_{0}} \sum_{x_{k} \in C_{m}}\left(\left\langle O_{i, m}\right\rangle-O_{i, k}\right)^{*}\left(\left\langle V_{i, m, s}\right\rangle-V_{i, k, s}\right), \\
\left\langle V_{i, m, s}\right)=\frac{1}{\left|C_{m}\right|} \sum_{x_{k}} \sum_{x_{k} \in C_{m}} V_{i, k, s}
\end{gathered}
$$

In formula (6), $Z$ is the learning rate; $k_{0}$ is the number of nodes in the output layer, namely, the total number of classes; $k$ is the number of quantum interval layers; $x_{k}: x_{k} \in C_{m}$ represents all samples belonging to the $C_{m}$ class.

Among them:

$$
V_{i, k, s}=O_{i, k, s} *\left(1-O_{i, k, s}\right) .
$$

In the formula: $O_{i, k, s}=\operatorname{sig}\left(U *\left(W^{\mathrm{T}} \mathrm{x}_{\mathrm{k}}-\theta_{\mathrm{s}}\right)\right)$ represents the output of the $s$ th quantum layer of the $i$ th hidden layer neuron when the input vector is $x_{k}(s=1,2, \cdots, n)$.

2.2. Caputo-Type Fractional Derivative Definition. In definition, $f(s)$ is a continuous function over $R$, for any $\beta>0$; the $\beta$-order Caputo-type derivative of $0, f(s)$ is defined as:

$$
D_{0, s}^{\beta} f(s)=D_{0, s}^{-(n-\beta)} \frac{d^{n}}{d t^{n}} f(s)=\frac{1}{\Gamma(n-\beta)} \int_{0}^{s}(s-\tau)^{\beta-1} f^{n}(\tau) \mathrm{d} \tau .
$$

The following corollary can be drawn:

(1) When $0<\beta<1, D_{0, s}^{\beta} f(s)=(1 /(\Gamma(1-\beta))) \int_{0}^{s}(s-\tau)^{\beta-1}$ $f(\tau) \mathrm{d} \tau$

(2) When $f(s)$ is a constant function, $D_{00}^{\beta} f(s)=0$

(3) $D^{-\beta} D^{\beta} f(s)=f(s)-\sum-f^{(m)}, \beta>0$,especially, when 0 $<\beta<1$, when $f(s)$
(4) is a one-dimensional function, $D^{-\beta} D^{\beta} f(s)=f(s)-f$ (0).

(5) If $\alpha$ and $\gamma$ are two constants, then $D^{-\beta}(\alpha f(s)+\gamma h(t))$ $=\alpha D^{-\beta} f(s)+\gamma D^{-\beta} h(t), \beta \geq 0$

2.3. Fractional-Order Quantum Neural Network Model. Suppose the following two conclusions are true:

(1) If vector $x=\left(x_{i}\right)$ and matrix $\bar{A}=\left(a_{i j}\right)$, we define the Euclidean norm $\|\mathrm{x}\|$ of vector $\mathbf{x}$ to be $\|x\|=\sum\left|x_{i}\right|$. The matrix norm of the matrix $\|\bar{A}\|$ is defined as $\| \bar{A}$ $\|=\max _{1 \leq i \leq n} \sum\left|a_{i j}\right|$. In this paper, we set $C=\|\bar{C}\|, A$ $=\|\bar{A}\|, B=\|\bar{B}\|$, and $M=\|\bar{M}\|$

(2) The excitation functions $F(x), G(x)$, and $H(x)$ of the fractional quantum neural network with mixed delay both satisfy the Lipschiz condition, that is, for any $u$ $, v \in R, u \neq v$, there exists a corresponding real number $F, G, H>0$, such that $\|F(u)-F(v)\| \leq F\|u-v\|$ , $\|G(u)-G(v)\| \leq G\|u-v\|$

The fractional quantum neural network model with mixed time delay is shown below:

$$
\left\{\begin{array}{l}
D^{\beta} x_{i}(t)=-c_{i} x_{i}(t)+\sum a_{i j} f_{j}\left(x_{j}(t)\right)+\sum b_{i j} g_{j}\left(x_{j}(t-\tau)\right)+\sum m_{i j} \int_{t-\sigma}^{t} h_{i}\left(x_{i}(\mu)\right) \mathrm{d} \mu+I_{i}, \\
x_{i}(t)=\psi_{i}(t), \quad t \in[-\gamma, 0), \gamma \in \max \{\tau, 0\},
\end{array}\right.
$$

is converted to:

$$
\left\{\begin{array}{l}
D^{\beta} x(t)=-\bar{C} x(t)+\bar{A} F(x(t))+\bar{B} G(x(t-\tau))+\bar{M} \int_{t-\sigma}^{t} H(\mu) \mathrm{d} \mu+I, \\
x(t)=\psi(t), \quad t \in[-\gamma, 0), \gamma \in \max \{\tau, \sigma\} .
\end{array}\right.
$$

Among them, $0<\beta<1,(i=1,2, \cdots, n), n$ represents the number of neurons in a fractional quantum neural network with mixed delay, and $x(t)=\left(x_{1}(t), x_{2}(t), \cdots, x_{n}(t)\right) \in R$ is the state vector of the neuron at time $t$.

$$
\begin{aligned}
F(x(t)) & =\left(f_{1}\left(x_{1}(t)\right), f_{2}\left(x_{2}(t)\right), \cdots, f_{n}\left(x_{n}(t)\right)\right), G(x(t)) \\
& =\left(g_{1}\left(x_{1}(t)\right), g_{2}\left(x_{2}(t)\right), \cdots, g_{n}\left(x_{n}(t)\right)\right)
\end{aligned}
$$

and $H(x(t))=\left(h_{1}\left(x_{1}(t)\right), h_{2}\left(x_{2}(t)\right), \cdots, h_{n}\left(x_{n}(t)\right)\right)^{T}$ are the activation function of fractional quantum neural network; $\bar{C}=\operatorname{diag}\left(c_{i}>0\right), \bar{A}=\left(a_{i i}\right), \bar{B}=\left(b_{i j}\right)$, and $\bar{M}=\left(m_{i j}\right)$ are all constant matrices; $c_{i}>0$ represents the rate of the isolated resting state of the first neuron in the fractional-order quantum neural network in the state of unconnected and without external additional voltage difference; $a_{j}, b_{i j}$, and $m_{i j}$ represent the weight of the connection between the $j$ th neuron and the $i$ th neuron; $\tau_{j}$ and $\sigma_{i}$ represent the transmission delay of the jth neuron along the axon; and $I=$ $\left(I_{1}(t), I_{2}(t), \cdots, I_{n}(t)\right)^{T}$ represents the external input and deviation of the neuron. 
Set the initial conditions of the system, usually assuming $\psi_{i}(s) \in C([-\gamma, 0] R), i \in N^{+}$, and the norm on $\mathrm{C}$ is defined as $\|\psi\|=\sup \|\psi(s)\|$.

Assume that $x(t)$ and $y(t)$ are two different solutions whose initial values of model (11) are $\psi \in C$ and $\phi \in C$, respectively, where $\psi(0)=\phi(0)=0$; let $\varphi=\psi-\varphi, x(t)=y(t)$ $=e(t)=\left(e_{1}(t), e_{2}(t), \cdots, e_{n}(t)\right)^{T}$ can be obtained:

$$
\begin{aligned}
D^{\beta} e(t)= & -C(t) \\
& +A(F(x(t))-F(y(t))) \\
& +B(G(x(t-\tau))-G(y(t-\tau))) \\
& +M \int_{t-\sigma}^{t}(H(x(\mu))-H(y(\mu))) \\
\cdot d \mu e(t)= & \varphi(t), \quad t \in[-\gamma, 0), \gamma \in \max \{\tau, \sigma\},
\end{aligned}
$$

where $\varphi \in C, \varphi(0)=0$ is the initial condition of model (12), $\|\varphi\|=\sup _{s \in[-\gamma, 0]}\|\varphi(s)\|$.

\section{Main Result}

Relevant definition:

Lemma 1. If $x(s) \in C^{n}[0,+\infty)$ and $n-1<\alpha, \beta<n \in Z^{+}$then

$$
\begin{aligned}
D^{-\alpha} D^{-\beta} x(s) & =D^{-(\alpha+\beta)} x(s), \quad \alpha, \beta>0, \\
D^{\beta} D^{-\beta} x(s) & =x(s), \quad \beta \geq 0, \\
D^{-\beta} D^{\beta} x(s) & =x(s)-\sum_{m=0}^{n-1} \frac{s^{m}}{m !} x^{(m)}, \quad \beta \geq 0 .
\end{aligned}
$$

Lemma 2 (Hölder inequality). Suppose that the real number $p, q>1$, and $p, q$ satisfies $(1 / p)+(1 / q)=1$, if $|f(\cdot)|^{p},|h(\cdot)|^{q}$ is a measurable function in space, and $f, g: E \longrightarrow R$ satisfies $\int_{E}|f(x)| d x<\infty, \int_{E}|g(x)| d x<\infty$, then $f(\cdot) h(\cdot)$ is also a measurable function and satisfies

$$
\int_{E}|f(x) h(x)| d x \leq\left(\int_{E}|f(x)|^{p} d x\right)^{1 / p}\left(\int_{E}|h(x)|^{q} d x\right)^{1 / q} .
$$

In particular, when $p=q=2$, it is the inequality that we usually see. That is

$$
\int_{E}|f(x) h(x)| d x \leq\left(\int_{E}|f(x)|^{2} d x\right)^{1 / 2}\left(\int_{E}|h(x)|^{2} d x\right)^{1 / 2} .
$$

Lemma 3. Let $k \in N, x_{1}, x_{2}, \cdots, x_{k}$ be a nonnegative real number, then it can be obtained for any

$$
\left(\sum_{i=1}^{k} x_{i}\right)^{\eta} \leq k^{\eta-1} \sum_{i=1}^{k}\left(x_{i}\right)^{\eta}
$$

Lemma 4 (Gronwall inequality). If $x(t), f(t), g(t) \geq 0$ is a continuous function on $[0, T), T<\infty$ and satisfies the following inequality

$$
x(t) \leq f(t)+\int_{0}^{t} g(\mu) x(\mu) d \mu, \quad t \in[0, T) .
$$

Then, we can get

$$
x(t) \leq f(t)+\int_{0}^{t} g(\mu) f(\mu) \exp \left\{\int_{\mu}^{t} g(v) d v\right\} d \mu, \quad t \in[0, T) .
$$

In special cases, if $f(t)$ is a nonincreasing function, you can get

$$
x(t) \leq f(t) \exp \left\{\int_{0}^{t} g(v) \mathrm{d} v\right\}, \quad t \in[0, T) .
$$

Definition 5. The initial time of the fractional quantum neural network system (11) with mixed delay is set to $t_{0}$. For any $\xi>0$, there are two constants $\delta$ and $T, 0<\delta<\xi, T>0$, so that for any $t \in J=\left[t_{0}, t_{0}+T\right]$, when $\left\|e\left(t_{0}\right)\right\|<\delta$ has $\|e(t)\|<\xi$, then the system (11) is called quasiuniformly stable.

Theorem 6. When the order $\beta \in[0.5,1)$ of the fractional quantum neural network system (11) with mixed delay is established, if the assumptions 2.3.1 and 2.3.2 are true and

$$
\begin{aligned}
& \sqrt{P+Q e^{2 t}+W(t) e^{(w(t)+2) t}\left(P \frac{1-e^{-(2+W(t)) t}}{2+W(t)}+Q \frac{1-e^{-W(t) t}}{W(t)}\right)} \\
& \quad<\frac{\xi}{\delta} t \in J,
\end{aligned}
$$

is true, where

$$
\begin{aligned}
& P=5-\frac{5 M^{2} H^{2} \gamma^{2} \Gamma(2 \beta-1)}{\Gamma^{2}(\beta) 4^{\beta}}, N=\frac{5 M^{2} H^{2}}{2 \beta \Gamma(\beta)}, \\
& Q=\frac{5 M^{2} H^{2} \gamma^{2} \Gamma(2 \beta-1)}{\Gamma^{2}(\beta) 4^{\beta}}+\frac{5 B^{2} G^{2} \Gamma(2 \beta-1)\left(1-e^{-2 \gamma}\right)}{\Gamma^{2}(\beta) 4^{\beta}}, \\
& L=\frac{10 \Gamma(2 \beta-1)\left[(C+A F)^{2}+B^{2} G^{2} e^{-2 \gamma}\right]}{\Gamma^{2}(\beta) 4^{\beta}} \\
& W(t)=L+N t^{2 \beta}\left(1-e^{-2 t}\right) .
\end{aligned}
$$

Then, the system (11) is quasiuniformly stable.

Proof. We set the initial time $t_{0}=0$ of the error system (12), the initial condition is $e_{0}=\varphi(0)$, and the expression of the solution of the error system can be obtained from Lemma 1 as 


$$
\begin{aligned}
e(t)= & \varphi(0)+D^{-\beta}[-\bar{C} e(t)+\bar{A}(F(x(t))-F(y(t))) \\
& \left.+\bar{B}(G(x(t-\tau))-G(y(t-\tau)))+\bar{M} \int_{t-\sigma}^{t}(H(x(\mu))-H(y(\mu))) \mathrm{d} \mu\right] \\
= & \varphi(0)+\frac{1}{\Gamma(\beta)} \int_{0}^{t}(t-\mu)^{\beta-1}[-\bar{C} e(\mu)+\bar{A}(F(x(\mu))-F(y(\mu))) \\
& \left.+\bar{B}(G(x(\mu-\tau))-G(y(\mu-\tau)))+\bar{M} \int_{t-\delta}^{t}(H(x(s))-H(y(s))) \mathrm{d} s\right] \mathrm{d} \mu .
\end{aligned}
$$

From the hypotheses 1 and 2 and the basic properties of the norm, we can get

$$
\begin{aligned}
\|e(t)\| \leq & \|\varphi(0)\|+\frac{1}{\Gamma(\beta)} \int_{0}^{t}(t-\mu)^{\beta-1}[C\|e(\mu)\|+A F\|e(\mu)\| \\
& \left.+B G\|e(\mu-\tau)\|+\int_{\mu-\sigma}^{\mu} M H\|e(s)\| \mathrm{d} s\right] \mathrm{d} \mu \\
\leq & \|\varphi(0)\|+\frac{1}{\Gamma(\beta)} \int_{0}^{t}(t-\mu)^{\beta-1}(C+A F)\|e(\mu)\| \mathrm{d} \mu \\
& +\frac{1}{\Gamma(\beta)} \int_{0}^{t}(t-\mu)^{\beta-1} B G\|e(\mu-\tau)\| \mathrm{d} \mu \\
& +\frac{1}{\Gamma(\beta)} \int_{0}^{t}(t-\mu)^{\beta-1}\left(\int_{-\sigma}^{t} M H\|e(s)\| \mathrm{d} s\right) \mathrm{d} \mu \\
\leq & \|\varphi\|+\frac{1}{\Gamma(\beta)} \int_{0}^{t}(t-\mu)^{\beta-1}(C+A F)\|e(\mu)\| \mathrm{d} \mu \\
& +\frac{1}{\Gamma(\beta)} \int_{0}^{t}(t-\mu)^{\beta-1} B G\|e(\mu-\tau)\| \mathrm{d} \mu \frac{M H t^{\beta}}{\beta \Gamma(\beta)} \int_{0}^{t}\|e(\mu)\| \mathrm{d} \mu \\
& +\frac{M H \sigma\|\varphi\|}{\Gamma(\beta)} \int_{0}^{t}(t-\mu)^{\beta-1} \mathrm{~d} \mu .
\end{aligned}
$$

According to the Cauchy-Schwartz inequality in Lemma 2, we know

$$
\begin{aligned}
\|e(t)\| \leq & \|\varphi\|+\frac{1}{\Gamma(\beta)}\left(\int_{0}^{t}(t-\mu)^{2 \beta-2} e^{2 \mu} \mathrm{d} \mu\right)^{1 / 2} \\
& \cdot\left(\int_{0}^{t}(C+A F)^{2}\|e(\mu)\|^{2} e^{-2 \mu} \mathrm{d} \mu\right)^{1 / 2} \\
& +\frac{1}{\Gamma(\beta)}\left(\int_{0}^{t}(t-\mu)^{2 \beta-2} e^{2 \mu} \mathrm{d} \mu\right)^{1 / 2} \\
& \cdot\left(\int_{0}^{t} B^{2} G^{2}\|e(\mu-\tau)\|^{2} e^{-2 \mu} \mathrm{d} \mu\right)^{1 / 2} \\
& +\frac{M H t^{\beta}}{\beta \Gamma(\beta)}\left(\int_{0}^{t} e^{2 \mu} \mathrm{d} \mu\right)^{1 / 2}\left(\int_{0}^{t}\|e(\mu)\|^{2} e^{-2 \mu} \mathrm{d} \mu\right)^{1 / 2} \\
& +\frac{M H \sigma\|\varphi\|}{\Gamma(\beta)}\left(\int_{0}^{t} e^{2 \mu}(t-\mu)^{2 \beta-2} \mathrm{~d} \mu\right)^{1 / 2}\left(\int_{0}^{t} e^{-2 \mu} \mathrm{d} \mu\right)^{1 / 2} .
\end{aligned}
$$

Bring

$$
\begin{aligned}
\int_{0}^{t}(t-\mu)^{2 \beta-2} e^{2 \mu} \mathrm{d} \mu & =\int_{0}^{t} z^{2 \beta-2} e^{2(t-z)} \mathrm{d} z=e^{2 t} \int_{0}^{t} z^{2 \beta-2} e^{-2 z} \mathrm{~d} z \\
& =\frac{e^{2 t}}{2^{2 \beta-1}} \int_{0}^{2 t} \mu^{2 \beta-2} e^{-\mu} \mathrm{d} \mu<\frac{2 e^{2 t}}{4^{\beta}} \Gamma(2 \beta-1),
\end{aligned}
$$

into Equation (24) to get

$$
\begin{aligned}
\|e(t)\| \leq & \|\varphi\|+\frac{1}{\Gamma(\beta)}\left(\frac{2 \Gamma(2 \beta-1) e^{2 t}}{4^{\beta}}\right)^{1 / 2} \\
& \cdot\left(\int_{0}^{t}(C+A F)^{2}\|e(\mu)\|^{2} e^{-2 \mu} \mathrm{d} \mu\right)^{1 / 2} \\
& +\frac{1}{\Gamma(\beta)}\left(\frac{2 \Gamma(2 \beta-1) e^{2 t}}{4^{\beta}}\right)^{1 / 2} \\
& \cdot\left(\int_{0}^{t} B^{2} G^{2}\|e(\mu-\tau)\|^{2} e^{-2 \mu} \mathrm{d} \mu\right)^{1 / 2} \\
& +\frac{M H t^{\beta}}{\beta \Gamma(\beta)}\left(\frac{e^{2 t}-1}{2}\right)^{1 / 2}\left(\int_{0}^{t}\|e(\mu)\|^{2} e^{-2 \mu} \mathrm{d} \mu\right)^{1 / 2} \\
& +\frac{M H \sigma\|\varphi\|}{\Gamma(\beta)}\left(\frac{2 \Gamma(2 \beta-1) e^{2 t}}{4^{\beta}}\right)^{1 / 2}\left(\frac{1-e^{-2 t}}{2}\right)^{1 / 2} .
\end{aligned}
$$

In Lemma 3 , let $k=5, \eta=2$, we can get

$$
\begin{aligned}
&\|e(t)\|^{2} \leq\left\{5-\frac{5 M^{2} H^{2} \gamma^{2} \Gamma(2 \beta-1)}{\Gamma^{2}(\beta) 4^{\beta}}+\left[\frac{5 M^{2} H^{2} \sigma^{2} \Gamma(2 \beta-1)}{\Gamma^{2}(\beta) 4^{\beta}}\right.\right. \\
&+\frac{\left.\left.5 B^{2} G^{2} \Gamma(2 \beta-1)\left(1-e^{2 \gamma}\right)\right] e^{2 t}\right\}\|\varphi\|^{2}}{\Gamma^{2}(\beta) 4^{\beta}} \\
&+\left\{\frac{10 \Gamma(2 \beta-1)\left[(C+A F)^{2}+B^{2} G^{2} e^{-2 \gamma}\right] e^{2 t}}{\Gamma^{2}(\beta) 4^{\beta}}\right. \\
&\left.+\frac{5 M^{2} H^{2} t^{2 \beta}\left(e^{2 t}-1\right)}{2 \beta \Gamma(\beta)}\right\} \int_{0}^{t}\|e(\mu)\|^{2} e^{-2 \mu} \mathrm{d} \mu, \\
& P=5- \frac{5 M^{2} H^{2} \gamma^{2} \Gamma(2 \beta-1)}{\Gamma^{2}(\beta) 4^{\beta}}, N=\frac{5 M^{2} H^{2}}{2 \beta \Gamma(\beta)}, \\
& Q=\frac{5 M^{2} H^{2} \gamma^{2} \Gamma(2 \beta-1)}{\Gamma^{2}(\beta) 4^{\beta}}+\frac{5 B^{2} G^{2} \Gamma(2 \beta-1)\left(1-e^{-2 \gamma}\right)}{\Gamma^{2}(\beta) 4^{\beta}}, \\
& L=\frac{10 \Gamma(2 \beta-1)\left[(C+A F)^{2}+B^{2} G^{2} e^{-2 \gamma}\right]}{\Gamma^{2}(\beta) 4^{\beta}},
\end{aligned}
$$

get

$$
\begin{aligned}
\|e(t)\|^{2} e^{-2 t} \leq & \left(P e^{-2 t}+Q\right)\|\varphi\|^{2}+\left(L+N t^{2 \beta}\right) \\
& \cdot\left(1-e^{-2 t}\right) \int_{0}^{t}\|e(\mu)\|^{2} e^{-2 \mu} \mathrm{d} \mu .
\end{aligned}
$$

Using Gronwall inequality and letting $W(t)=L+N t^{2 \beta}$ $\left(1-e^{-2 t}\right)$, get

$$
\begin{aligned}
\|e(t)\|^{2} e^{-2 t} \leq & {\left[P e^{-2 t}+Q+W(t) e^{\operatorname{tw}(t)}\left(P \frac{1-e^{-(2+W(t)) t}}{2+W(t)}+Q \frac{1-e^{-W(t) t}}{W(t)}\right)\right] } \\
& \cdot\|\varphi\|^{2},
\end{aligned}
$$




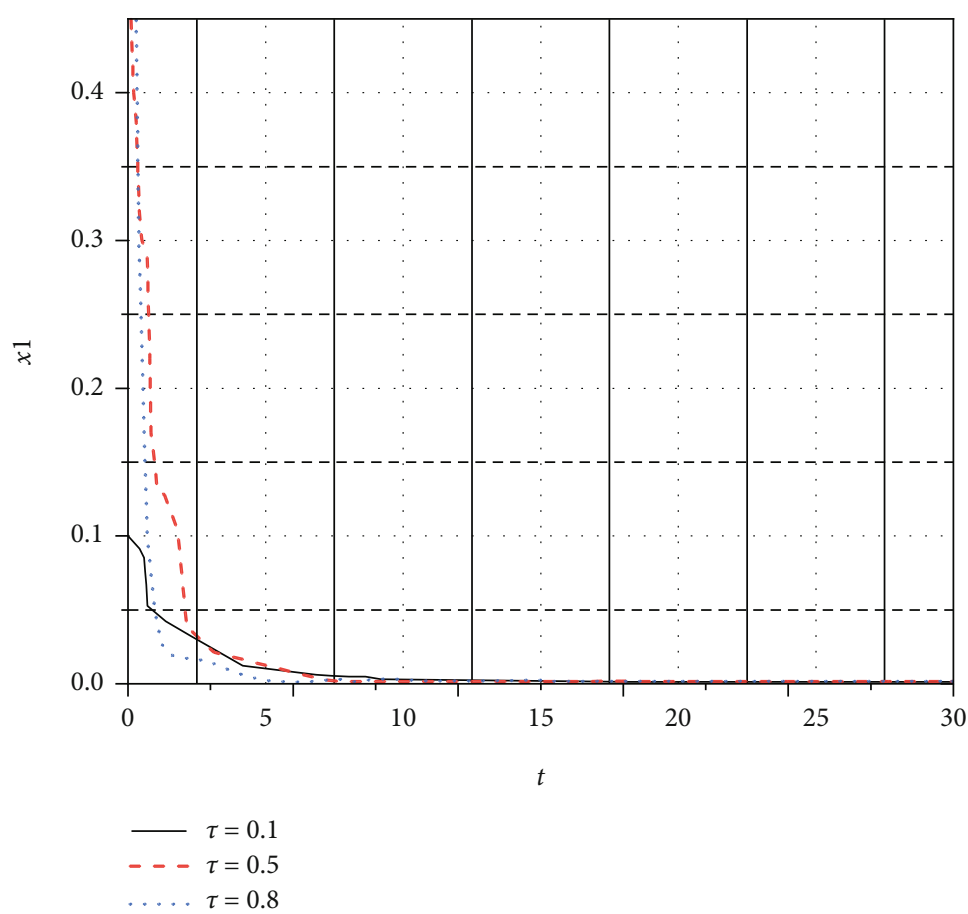

FIgURE 2: The trajectory graph of $x_{1}$ for different $\tau$.

$$
\begin{aligned}
\|e(t)\|^{2} \leq & {\left[P+Q e^{2 t}+W(t) e^{(w(t)+2) t}\left(P \frac{1-e^{-(2+W(t)) t}}{2+W(t)}+Q \frac{1-e^{-W(t) t}}{W(t)}\right)\right] } \\
& \cdot\|\varphi\|^{2},
\end{aligned}
$$

that is

$$
\begin{aligned}
\|e(t)\| \leq & \sqrt{P+Q e^{2 t}+W(t) e^{(w(t)+2) t}\left(P \frac{1-e^{-(2+W(t)) t}}{2+W(t)}+Q \frac{1-e^{-W(t) t}}{W(t)}\right)} \\
& \cdot\|\varphi\| .
\end{aligned}
$$

It can be seen that when $\|\varphi\|<\delta,\|e(t)\|<\xi$ is easy to know from Theorem 6. From Definition 5, it can be concluded that the fractional quantum neural system (11) with mixed time delay is quasiuniformly stable.

Theorem 7. If the order $\beta \in(0,0.5)$ of fractional-order quantum neural network system (11) with mixed delay is true, assuming that 1 and 2 are true and

$$
\sqrt[q]{\tilde{P}+\tilde{Q} e^{q t}+\frac{\tilde{W}(t) \tilde{P}\left(e^{(\tilde{w}(t)+q) t}-1\right)}{q+\tilde{W}(t)}+\frac{\tilde{W}(t) \tilde{Q} e^{(\tilde{w}(t)+q) t}\left(1-e^{-W(t) t}\right)}{\tilde{W}(t)}}<\frac{\xi}{\delta} t \in J
$$

is true, where

$$
\begin{aligned}
& \tilde{P}=5^{q-1}, \tilde{Q}=\frac{5^{q-1} B^{q} G^{q} \tilde{E}\left(1-e^{-q \gamma}\right)}{q}+5^{q-1} M^{q} H^{q} \gamma^{q} \tilde{E}, \\
& \tilde{L}=5^{q-1} \tilde{E}(C+A F)^{q}+5^{q-1} B^{q} G^{q} e^{-q \gamma} \tilde{E}, \\
& \tilde{E}=\left[\frac{\Gamma(p \beta-p+1)}{\Gamma^{p}(\beta) p^{p \beta-p+1}}\right]^{q / p}, \tilde{W}(t)=L+\tilde{N} t^{q \beta}, \tilde{N}=\frac{5^{q-1} M^{q} H^{q}}{\beta^{q} \Gamma^{q}(\beta)},
\end{aligned}
$$

then the system (11) is quasiuniformly stable.

Proof from Theorem 6, we get

$$
\begin{aligned}
\|e(t)\| \leq & \|\varphi\|+\frac{1}{\Gamma(\beta)} \int_{0}^{t}(t-\mu)^{\beta-1}(C+A F)\|e(\mu)\| \mathrm{d} \mu \\
& +\frac{1}{\Gamma(\beta)} \int_{0}^{t}(t-\mu)^{\beta-1} B G\|e(\mu-\tau)\| \mathrm{d} \mu \\
& +\frac{1}{\Gamma(\beta)} \int_{0}^{t}(t-\mu)^{\beta-1}\left(\int_{\mu-\sigma}^{\mu} M H\|e(s)\| \mathrm{d} s\right) \mathrm{d} \mu,
\end{aligned}
$$

let $p=1+\beta$ and $p=1+(1 / \beta)$, obviously $p, q>1$, from Hölder's inequality we can get

$$
\begin{aligned}
\|e(t)\| \leq & \|\varphi\|+\frac{1}{\Gamma(\beta)}\left(\int_{0}^{t}(t-\mu)^{p \beta-p} e^{p \mu} \mathrm{d} \mu\right)^{1 / p} \\
& \cdot\left(\int_{0}^{t}(C+A F)^{q}\|e(\mu)\|^{q} e^{-q \mu} \mathrm{d} \mu\right)^{1 / q}+\frac{1}{\Gamma(\beta)} \\
& \cdot\left(\int_{0}^{t}(t-\mu)^{p \beta-p} e^{p \mu} \mathrm{d} \mu\right)^{1 / p}\left(\int_{0}^{t} B^{q} G^{q}\|e(\mu-\tau)\|^{q} e^{-q \mu} \mathrm{d} \mu\right)^{1 / q} \\
& +\frac{M H t^{\beta} e^{p t}}{\beta \Gamma(\beta)}\left(\int_{0}^{t}\|e(\mu)\|^{q} e^{-q \mu} \mathrm{d} \mu\right)^{1 / q}+\frac{M H \sigma\|\varphi\|}{\Gamma(\beta)} \\
& \cdot\left(\int_{0}^{t} e^{p \mu}(t-\mu)^{p \beta-p} \mathrm{~d} \mu\right)^{1 / q},
\end{aligned}
$$




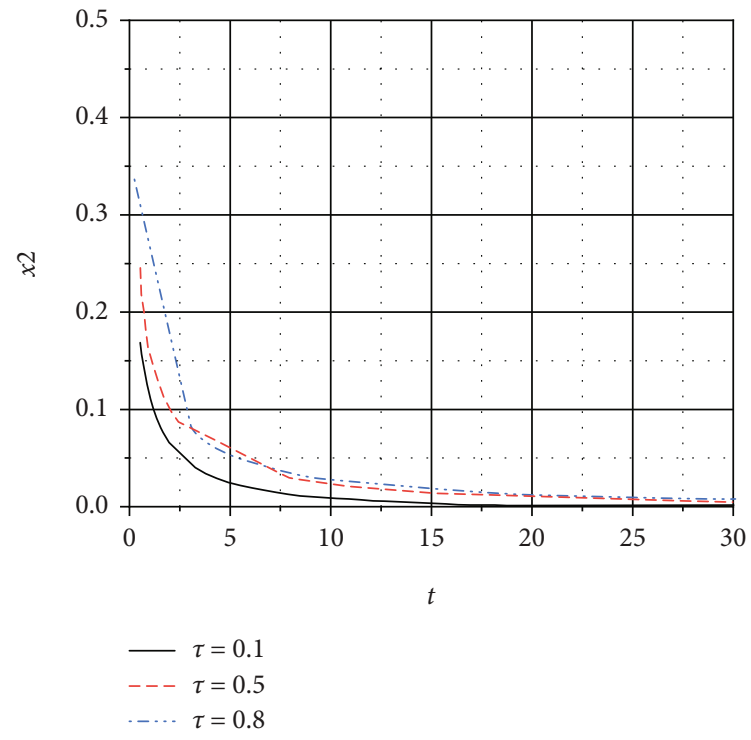

Figure 3: The trajectory graph of $x_{2}$ for different $\tau$.

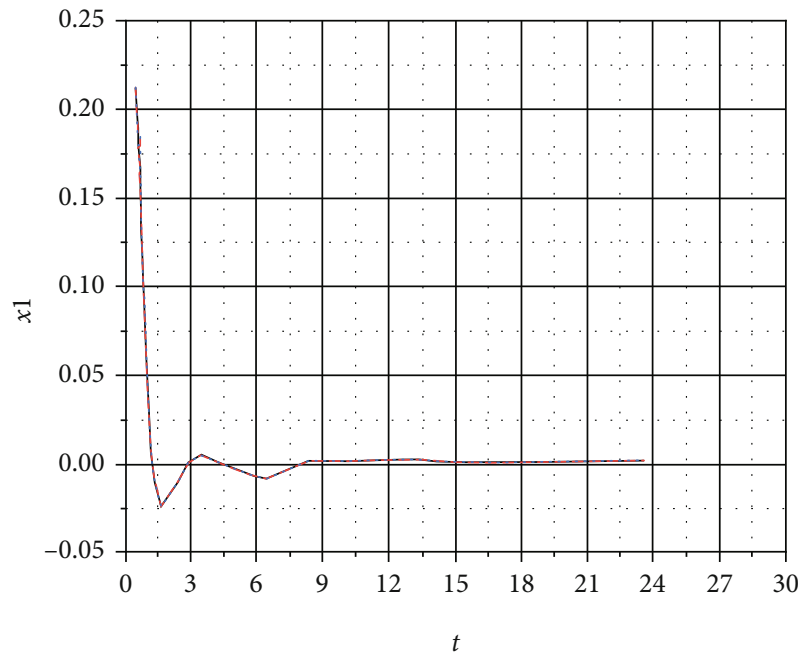

$$
\begin{aligned}
-\sigma & =0.05 \\
---\sigma & =0.2 \\
\cdots \cdots & \sigma=0.3
\end{aligned}
$$

FIGURE 4: The trajectory graph of $x_{1}$ for different $\sigma$.

because

$$
\begin{aligned}
\int_{0}^{t}(t-\mu)^{p \beta-p} e^{p \mu} \mathrm{d} \mu & =\int_{0}^{t} z^{p \beta-p} e^{p(t-z)} \mathrm{d} z=e^{p t} \int_{0}^{t} z^{p \beta-p} e^{-p z} \mathrm{~d} z \\
& =\frac{e^{p t}}{p^{p \beta-p+1}} \int_{0}^{p t} \mu^{p \beta-p} e^{-\mu} \mathrm{d} \mu<\frac{e^{p t}}{p^{p \beta-p+1}} \Gamma(p \beta-p+1),
\end{aligned}
$$

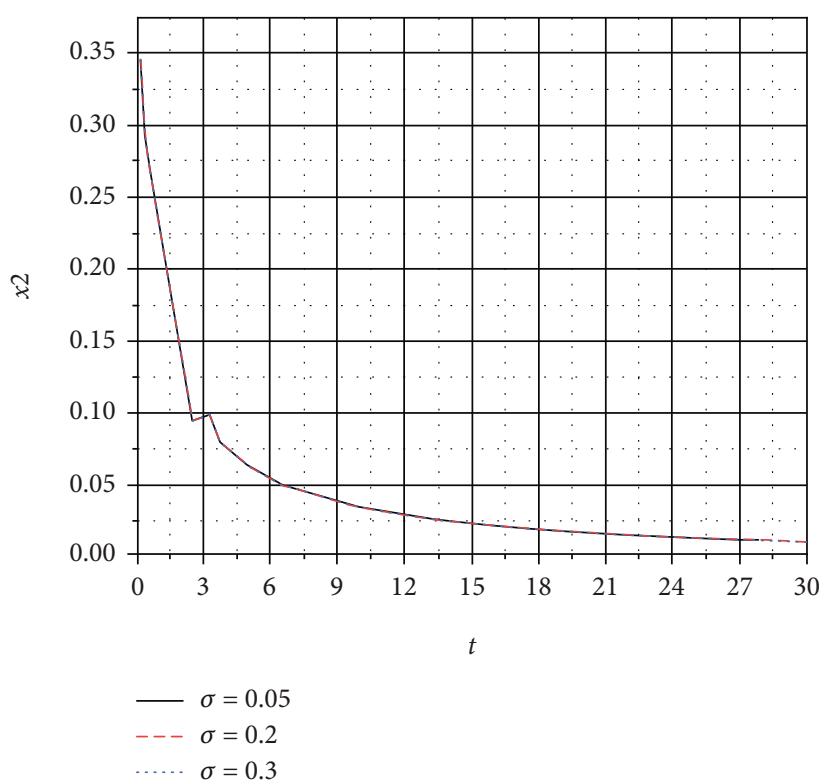

FIGURE 5: The trajectory graph of $x_{2}$ for different $\sigma$.

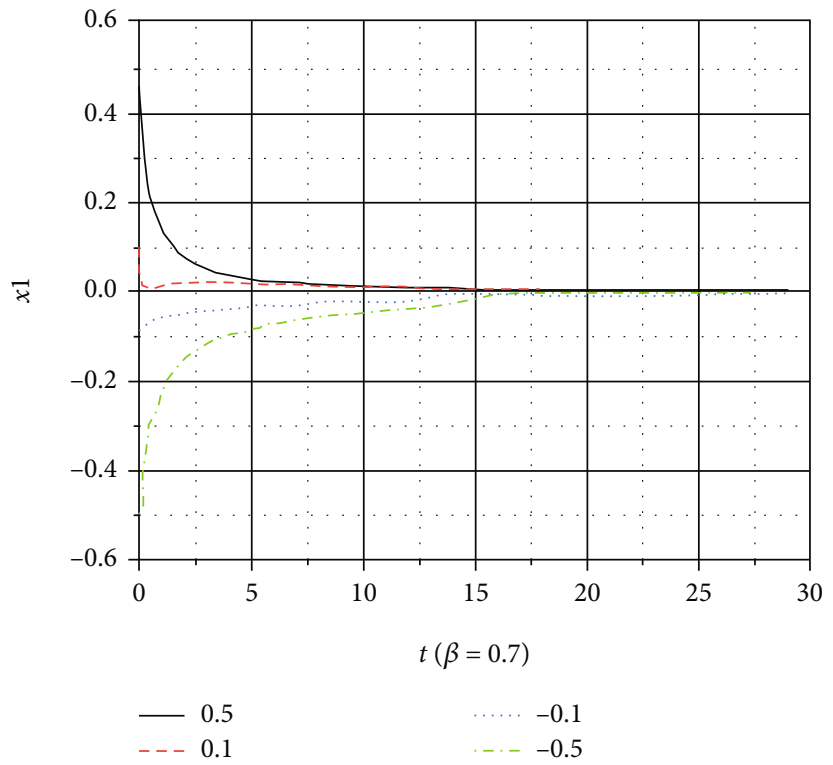

FIgURE 6: For the trajectory graph of different initial values $x_{1}$.

$$
\begin{aligned}
\|e(t)\| \leq & \|\varphi\|+\left(\frac{e^{p t} \Gamma(p \beta-p+1)}{\Gamma^{p}(\beta) p^{p \beta-p+1}}\right)^{1 / p}\left(\int_{0}^{t}(C+A F)^{q}\|e(\mu)\|^{q} e^{-q \mu} \mathrm{d} \mu\right)^{1 / q} \\
& +\left(\frac{e^{p t} \Gamma(p \beta-p+1)}{\Gamma^{p}(\beta) p^{p \beta-p+1}}\right)^{1 / p}\left(\int_{0}^{t} B^{q} G^{q}\|e(\mu-\tau)\|^{q} e^{-q \mu} \mathrm{d} \mu\right)^{1 / q} \\
& +\frac{M H t^{\beta} e^{p t}}{\beta \Gamma(\beta)}\left(\int_{0}^{t}\|e(\mu)\|^{q} e^{-q \mu} \mathrm{d} \mu\right)^{1 / q}+M H \sigma\|\varphi\| \\
& \cdot\left(\frac{e^{p t} \Gamma(p \beta-p+1)}{\Gamma^{p}(\beta) p^{p \beta-p+1}}\right)^{1 / p} .
\end{aligned}
$$




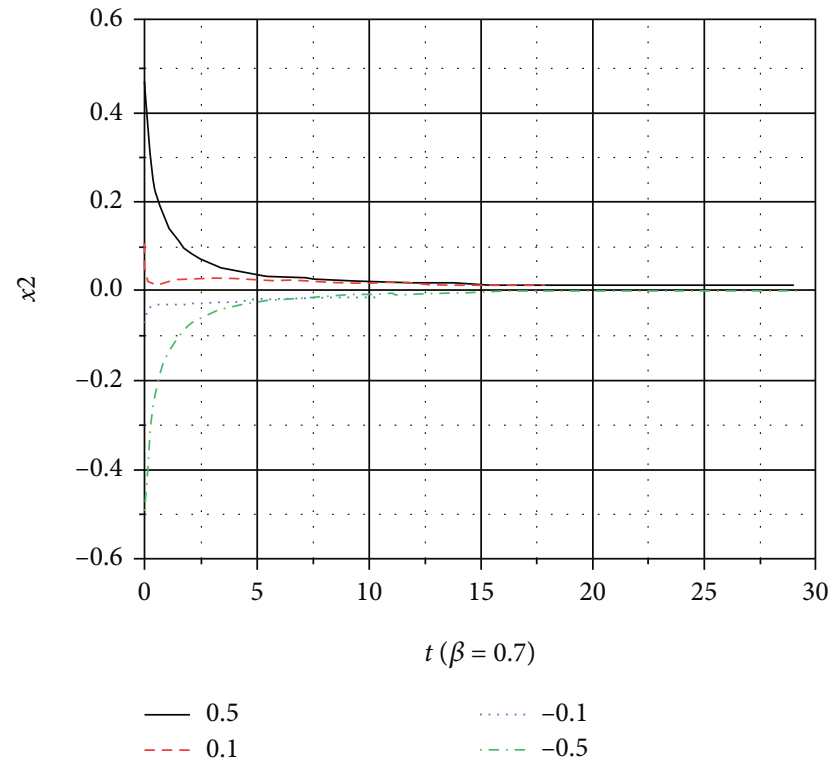

FIgURE 7: For the trajectory graph of different initial values $x_{2}$.

In Lemma 3, let $k=5, \eta=q$, we can get

$$
\begin{aligned}
\|e(t)\|^{q} \leq & 5^{q-1}\|\varphi\|^{q}+5^{q-1}\left(\frac{e^{p t} \Gamma(p \beta-p+1)}{\Gamma^{p}(\beta) p^{p \beta-p+1}}\right)^{q / p} \\
& \cdot \int_{0}^{t}(C+A F)^{q}\|e(\mu)\|^{q} e^{-q \mu} \mathrm{d} \mu+5^{q-1}\left(\frac{e^{p t} \Gamma(p \beta-p+1)}{\Gamma^{p}(\beta) p^{p \beta-p+1}}\right)^{q / p} \\
& \cdot \int_{0}^{t} B^{q} G^{q}\|e(\mu-\tau)\|^{q} e^{-q \mu} \mathrm{d} \mu+\frac{5^{q-1} M^{q} H^{q} t^{q \beta} e^{p t}}{\beta^{q} \Gamma^{q}(\beta)} \\
& \cdot \int_{0}^{t}\|e(\mu)\|^{q} e^{-q \mu} \mathrm{d} \mu+5^{q-1} M^{q} H^{q} \sigma^{q}\|\varphi\|^{q} \\
& \cdot\left(\frac{e^{p t} \Gamma(p \beta-p+1)}{\Gamma^{p}(\beta) p^{p \beta-p+1}}\right)^{q / p} .
\end{aligned}
$$

Let

$$
\tilde{E}=\left[\frac{\Gamma(p \beta-p+1)}{\Gamma^{p}(\beta) p^{p \beta-p+1}}\right]^{q / p},
$$

get

$$
\begin{aligned}
\|e(t)\|^{q} \leq & \left(5^{q-1}+5^{q-1} M^{q} H^{q} \gamma^{q} \tilde{E} e^{q t}+\frac{5^{q-1} B^{q} G^{q} \tilde{E}\left(1-e^{-q \gamma}\right) e^{q t}}{q}\right)\|\varphi\|^{q} \\
& +\left[5^{q-1} \tilde{E}(C+A F)^{q} e^{q t}+\frac{5^{q-1} M^{q} H^{q} t^{q \beta} e^{q t}}{\beta^{q} \Gamma^{q}(\beta)}+5^{q-1} B^{q} G^{q} e^{-q \gamma} \tilde{E} e^{q t}\right] \\
& \cdot \int_{0}^{t}\|e(\mu)\|^{q} e^{-q \mu} \mathrm{d} \mu,
\end{aligned}
$$

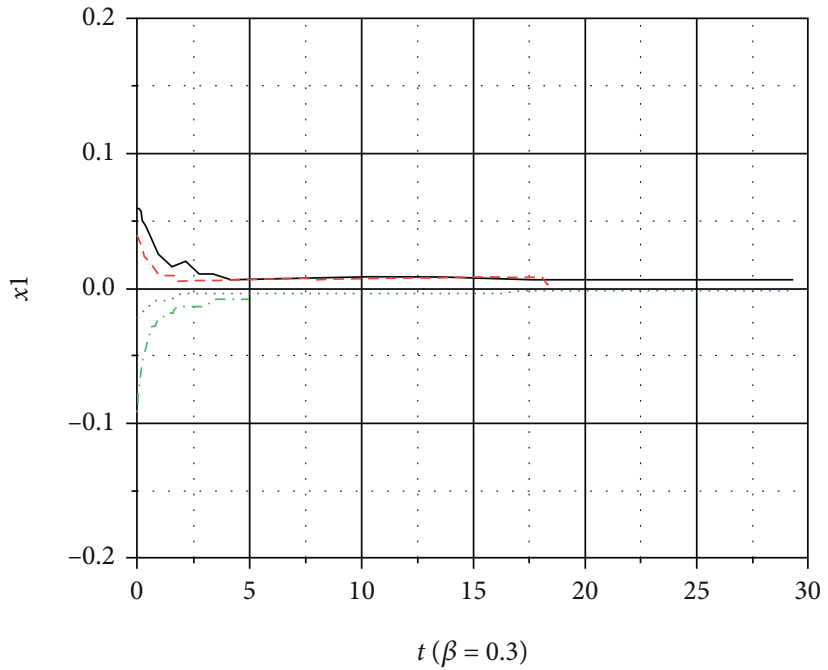

FIgURE 8: For the trajectory graph of different initial values $x_{1}$.

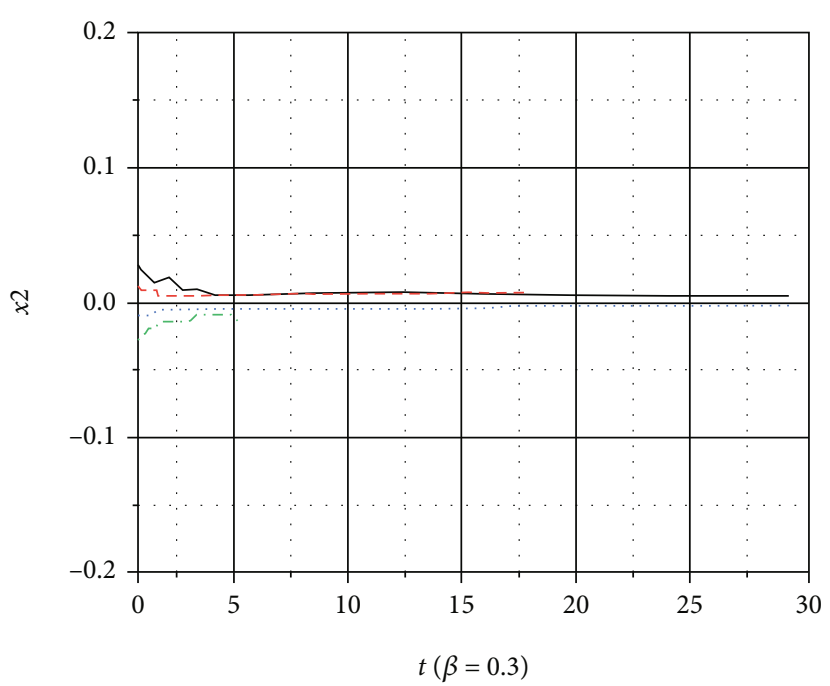

FIgURE 9: For the trajectory graph of different initial values $x_{2}$.

then let

$$
\begin{aligned}
& \tilde{P}=5^{q-1}, \tilde{N}=\frac{5^{q-1} M^{q} H^{q}}{\beta^{q} \Gamma^{q}(\beta)}, \\
& \tilde{Q}=\frac{5^{q-1} B^{q} G^{q} \tilde{E}\left(1-e^{-q \gamma}\right)}{q}+5^{q-1} M^{q} H^{q} \gamma^{q} \tilde{E}, \\
& \tilde{L}=5^{q-1} \tilde{E}(C+A F)^{q}+5^{q-1} B^{q} G^{q} e^{-q \gamma} \tilde{E},
\end{aligned}
$$

then

$$
\|e(t)\|^{q} e^{-q t} \leq\left(\tilde{P} e^{-q t}+Q\right)\|\varphi\|^{q}+\left(\tilde{L}+\tilde{N} t^{q \beta}\right) \int_{0}^{t}\|e(\mu)\|^{q} e^{-q \mu} \mathrm{d} \mu .
$$

Use Gronwall inequality and make $\tilde{W}(t)=L+\tilde{N} t^{q \beta}$, and get 


$$
\begin{aligned}
\|e(t)\|^{q} \leq & {\left[\tilde{P}+\tilde{Q} e^{q t}+\frac{\tilde{W}(t) \tilde{P}\left(e^{(\tilde{w}(t)+q) t}-1\right)}{q+\tilde{W}(t)}+\frac{\tilde{W}(t) \tilde{Q} e^{(\tilde{w}(t)+q) t}\left(1-e^{-W(t) t}\right)}{\tilde{W}(t)}\right] } \\
& \cdot\|\varphi\|^{q},
\end{aligned}
$$

which is

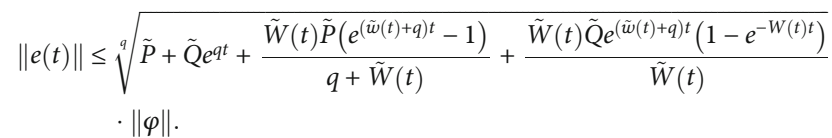

It can be seen that when $\|\varphi\|<\delta$, it is easy to know $\|e(t)\|<\xi$ from Theorem 6. From Definition 5, it can be obtained that the fractional quantum neural system (11) with mixed time delay is quasiuniformly stable.

\section{Illustration}

In this part, we give a specific example to verify the validity and applicability of the given results.

$$
\left\{\begin{array}{l}
D^{\beta}\left(x_{2}(t)\right)=-0.1 x_{2}(t)+0.1 f_{1}\left(x_{1}(t)\right)-0.2 f_{2}\left(x_{2}(t)\right)-0.2 g_{1}\left(x_{1}(t-\tau)\right)-0 . \lg _{2}\left(x_{2}(t-\tau)\right)+\int_{t-\sigma}^{t}\left[0.1 h_{1}\left(x_{1}(\mu)\right)+0.2 h_{2}\left(x_{2}(\mu)\right)\right] d \mu \\
D^{\beta}\left(x_{1}(t)\right)=-0.1 x_{1}(t)+0.2 f_{1}\left(x_{1}(t)\right)-0.1 f_{2}\left(x_{2}(t)\right)-0.5 g_{1}\left(x_{1}(t-\tau)\right)-0 . \lg _{2}\left(x_{2}(t-\tau)\right)+\int_{t-\sigma}^{t}\left[0.4 h_{1}\left(x_{1}(\mu)\right)-0.1 h_{2}\left(x_{2}(\mu)\right)\right] d \mu
\end{array}\right.
$$

The activation function in the above formula is: $f_{i}\left(x_{i}(t)\right)=g_{i}\left(x_{i}(t)\right)=h_{i}\left(x_{i}(t)\right)=\operatorname{sigmoid}(x), i=(1,2 \cdots)$, $F=H=G=1$.

$$
\text { By } \quad \bar{B}=\left(\begin{array}{ll}
-0.5 & -0.1 \\
-0.2 & -0.1
\end{array}\right), \quad \bar{A}=\left(\begin{array}{cc}
0.2 & -0.1 \\
0.1 & -0.2
\end{array}\right), \quad \bar{C}=
$$$$
\left(\begin{array}{cc}
0.1 & 0 \\
0 & 0.1
\end{array}\right) \text {, and } \bar{M}=\left(\begin{array}{cc}
0.4 & -0.1 \\
0.1 & 0.2
\end{array}\right) \text {, inferred } B=0.7, A
$$
$=0.3, C=0.1$, and $M=0.5$.

In this experiment, the experimental data show that by controlling the corresponding parameters, we can study the influence of another parameter on the trajectory of $x_{1}$ and $x_{2}$ with different initial values. We set the parameters $\delta=$ $0.1, \sigma=0.05, t_{0}=0$. Set the parameters when $\beta=0.7$, find $L$ $=2.7994, \quad Q=0.2278, \quad N=0.6878, \quad W(t)=3.0884, \quad P=$ 4.9938, and find $T=0.6690$ from the following inequality:

$$
\sqrt{P+Q e^{2 t}+W(t) e^{(W(t)+2) t}\left(P \frac{1-e^{-(W(t)+2) t}}{W(t)+2}+Q \frac{1-e^{-W(t) t}}{W(t)}\right)}<\frac{\varepsilon}{\delta} .
$$

When $\quad \beta=0.3, \quad$ find $\quad \tilde{L}=83.7659, \tilde{Q}=9.2269, \tilde{N}=$ 16.9440, $\tilde{W}(t)=84.1261, \tilde{P}=213.7471, \tilde{E}=2.4949$. Find $T$ $=0.0522$ from the following inequality.

Figure 2 is for $\sigma=0.1, \beta=0.7$, and $x_{1}(t)=0.5$. For different $\tau$ values $(\tau=0.1,0.5,0.8)$, the corresponding trajectory of $x_{1}$. Figure 3 is for $\sigma=0.1, \beta=0.7$, and $x_{2}(t)=0.5$. For different $\tau$ values $(\tau=0.1,0.5,0.8)$, the corresponding trajectory of $x_{2}$. It can be seen that the state trajectories of $x_{1}$ and $x_{2}$ converge to the equilibrium point.

Figure 4 shows the trajectory of $x_{1}$ for $\tau=1, \beta=0.7$, and the initial value $x_{1}(t)=0.5$, for different values of $\sigma=0.05,0.2$, 0.3 . Figure 5 shows the trajectory of $x_{1}$ for $\tau=1, \beta=0.7$, the initial value $x_{2}(t)=0.5$, for different values of $\sigma=0.05,0.2,0.3$ the trajectory of $x_{2}$. It can be seen that the state trajectories of $x_{1}$ and $x_{2}$ converge to the equilibrium point.

Figure 6 shows the trajectory of $x_{1}$ when $\beta=0.7, \sigma=0.05$, and $\tau=0.1$, and the initial value $x_{1}(t)$ takes different values. Figure 7 shows the trajectory of $x_{2}$ when $\beta=0.7, \sigma=0.05$, and $\tau=0.1$, and the initial value $x_{2}(t)$ takes different values. It can be seen that the state trajectories of $x_{1}$ and $x_{2}$ converge to the equilibrium point.

Figure 8 shows the trajectory of $x_{1}$ when $\beta=0.3, \sigma=0.05$, $\tau=0.1$, and the initial value $x_{1}(t)$ takes different values. Figure 9 shows the trajectory of $x_{2}$ when $\beta=0.3, \sigma=0.05$, and $\tau=0.1$, and the initial value $x_{2}(t)$ takes different values. It can be seen that the state trajectories of $\mathrm{x}_{1}$ and $x_{2}$ converge to the equilibrium point.

\section{Conclusions}

This paper uses the linear superposition of multilayer activation functions, uses learning algorithms to adjust quantum intervals and other operations to quantize the neural network, and proposes a quantum neural network model with multilayer activation functions. On this basis, the quasiuniform stability of fractional quantum neural networks with mixed time delays is studied. When $\beta$ belongs to different ranges, the sufficient conditions for the quasiuniform stability of the fractional quantum neural network system with mixed time delay are, respectively, discussed. Using the corresponding theorem, the proof of the theoretical result is given. Finally, through numerical simulation, the feasibility of the conclusions obtained in this paper is verified.

\section{Data Availability}

We did not use the data set in the research of this article. 


\section{Conflicts of Interest}

The authors declare that they have no conflicts of interest.

\section{Acknowledgments}

This work received support from the National Natural Science Foundation of China (Nos. 61772295, 61572270, and 61173056), the PHD Foundation of Chongqing Normal University (No. 19XLB003), the Science and Technology Research Program of Chongqing Municipal Education Commission (Grant no. KJZD-M202000501), and Chongqing Technology Innovation and Application Development Special General Project (cstc2020jscx-lyjsAX0002).

\section{References}

[1] C. J. Zuñiga Aguilar, J. Gómez-Aguilar, V. Alvarado-Martínez, and H. Romero-Ugalde, "Fractional order neural networks for system identification," Chaos, Solitons \& Fractals, vol. 130, article 109444, 2020.

[2] D. Sheng, Y. Wei, Y. Chen, and Y. Wang, "Convolutional neural networks with fractional order gradient method," Neurocomputing, vol. 408, pp. 42-50, 2020.

[3] J. Wang, Y. Wen, Y. Gou, Z. Ye, and H. Chen, "Fractionalorder gradient descent learning of BP neural networks with Caputo derivative," Neural Networks, vol. 89, pp. 19-30, 2017.

[4] A. Boroomand and M. B. Menhaj, "Fractional-order Hopfield neural networks," in Advances in Neuro-Information Processing. ICONIP 2008, M. Köppen, N. Kasabov, and G. Coghill, Eds., vol. 5506 of Lecture Notes in Computer Science, pp. 883-890, Springer, Berlin, Heidelberg, 2008.

[5] L. Zhang and Y. Yang, "Different impulsive effects on synchronization of fractional-order memristive BAM neural networks," Nonlinear Dynamics, vol. 93, no. 2, pp. 233-250, 2018.

[6] Y. Gu, Y. Yu, and H. Wang, "Synchronization-based parameter estimation of fractional-order neural networks," Physica A: Statistical Mechanics and its Applications, vol. 483, pp. 351-361, 2017.

[7] L. Chen, C. Liu, R. Wu, Y. He, and Y. Chai, "Finite-time stability criteria for a class of fractional-order neural networks with delay," Neural Computing and Applications, vol. 27, no. 3, pp. 549-556, 2016.

[8] Y. Xingyu and J. Lu, "Synchronization of fractional order memristor-based inertial neural networks with time delay," in 2020 Chinese Control And Decision Conference (CCDC), Hefei, China, 2020.

[9] W. Zhang, J. Cao, D. Chen, and F. Alsaadi, "Synchronization in fractional-order complex-valued delayed neural networks," Entropy, vol. 20, no. 1, p. 54, 2018.

[10] L. Kexue, P. Jigen, and G. Jinghuai, "A comment on " $\alpha$-stability and $\alpha$-synchronization for fractional-order neural networks"," Neural Networks, vol. 48, pp. 207-208, 2013.

[11] H. Liu, S. Li, H. Wang, Y. Huo, and J. Luo, “Adaptive synchronization for a class of uncertain fractional-order neural networks," Entropy, vol. 17, no. 12, pp. 7185-7200, 2015.

[12] T. Hu, X. Zhang, and S. Zhong, "Global asymptotic synchronization of nonidentical fractional-order neural networks," Neurocomputing, vol. 313, pp. 39-46, 2018.
[13] D. Sierociuk, G. Sarwas, and A. Dzieliński, "Discrete fractional order artificial neural network," Acta Mechanica et Automatica, vol. 5, pp. 128-132, 2011.

[14] X. Huang, Z. Zhao, Z. Wang, and Y. Li, "Chaos and hyperchaos in fractional-order cellular neural networks," Neurocomputing, vol. 94, pp. 13-21, 2012.

[15] C. Song and J. Cao, "Dynamics in fractional-order neural networks," Neurocomputing, vol. 142, pp. 494-498, 2014.

[16] I. Batiha, R. Albadarneh, S. M. Momani, and I. H. Jebril, "Dynamics analysis of fractional-order Hopfield neural networks," International Journal of Biomathematics, vol. 13, no. 8, article 2050083, 2020.

[17] M.-R. Chen, B.-P. Chen, G.-Q. Zeng, K.-D. Lu, and P. Chu, "An adaptive fractional-order BP neural network based on extremal optimization for handwritten digits recognition," Neurocomputing, vol. 391, pp. 260-272, 2020.

[18] M. Wu, J. Zhang, Z. Huang, X. Li, and Y. Dong, "Numerical solutions of wavelet neural networks for fractional differential equations," Mathematicsl Methods in the Applied Sciences, pp. $1-14,2021$.

[19] C. Lu and X. Ding, "Periodic solutions and stationary distribution for a stochastic predator-prey system with impulsive perturbations," Applied Mathematics and Computation, vol. 350, pp. 313-322, 2019.

[20] Z. Aslipour and A. Yazdizadeh, "Identification of nonlinear systems using adaptive variable-order fractional neural networks (case study: a wind turbine with practical results)," Engineering Applications of Artificial Intelligence, vol. 85, pp. 462473, 2019.

[21] C. Lu, G. Sun, and Y. Zhang, "Stationary distribution and extinction of a multi-stage HIV model with nonlinear stochastic perturbation," Journal of Applied Mathematics and Computing, pp. 1-23, 2021.

[22] G. Rajchakit, P. Chanthorn, M. Niezabitowski, R. Raja, D. Baleanu, and A. Pratap, "Impulsive effects on stability and passivity analysis of memristor-based fractional-order competitive neural networks," Neurocomputing, vol. 417, pp. 290-301, 2020.

[23] P. Chanthorn, G. Rajchakit, S. Ramalingam, C. P. Lim, and R. Ramachandran, "Robust dissipativity analysis of Hopfieldtype complex-valued neural networks with time-varying delays and linear fractional uncertainties," Mathematics, vol. 8, no. 4, p. 595, 2020.

[24] U. Humphries, G. Rajchakit, P. Kaewmesri et al., "Global stability analysis of fractional-order quaternion-valued bidirectional associative memory neural networks," Mathematics, vol. 8, no. 5, p. 801, 2020.

[25] G. Rajchakit, P. Chanthorn, P. Kaewmesri, R. Sriraman, and C. P. Lim, "Global Mittag-Leffler stability and stabilization analysis of fractional-order quaternion-valued memristive neural networks," Mathematics, vol. 8, no. 3, p. 422, 2020.

[26] S. M. A. Pahnehkolaei, A. Alfi, and J. T. Machado, "Stability analysis of fractional quaternion-valued leaky integrator echo state neural networks with multiple time-varying delays," Neurocomputing, vol. 331, pp. 388-402, 2019.

[27] S. M. A. Pahnehkolaei, A. Alfi, and J. T. Machado, "Delay independent robust stability analysis of delayed fractional quaternion-valued leaky integrator echo state neural networks with QUAD condition," Applied Mathematics and Computation, vol. 359, pp. 278-293, 2019. 
[28] S. M. Abedi Pahnehkolaei, A. Alfi, and J. A. T. Machado, "Uniform stability of fractional order leaky integrator echo state neural network with multiple time delays," Information Sciences, vol. 418-419, pp. 703-716, 2017.

[29] R. Rakkiyappan, J. Cao, and G. Velmurugan, "Existence and uniform stability analysis of fractional-order complex-valued neural networks with time delays," IEEE Transactions on Neural Networks and Learning Systems, vol. 26, no. 1, pp. 84-97, 2015.

[30] W. Ran-Chao, H. Xin-Dong, and C. Li-Ping, "Finite-time stability of fractional-order neural networks with delay," Communications in Theoretical Physics, vol. 60, p. 189, 2013.

[31] S. Zhang, Y. Yu, and Q. Wang, "Stability analysis of fractionalorder Hopfield neural networks with discontinuous activation functions," Neurocomputing, vol. 171, pp. 1075-1084, 2016.

[32] H. Wu, X. Zhang, S. Xue, and P. Niu, "Quasi-uniform stability of Caputo-type fractional-order neural networks with mixed delay," International Journal of Machine Learning and Cybernetics, vol. 8, no. 5, pp. 1501-1511, 2017.

[33] A. Alofi, J. Cao, A. Elaiw, and A. Al-Mazrooei, "Delay-dependent stability criterion of Caputo fractional neural networks with distributed delay," Discrete Dynamics in Nature and Society, vol. 2014, 6 pages, 2014

[34] G. Purushothaman and N. B. Karayiannis, "Quantum neural networks (QNNS): inherently fuzzy feedforward neural networks," IEEE Transactions on Neural Networks, vol. 8, no. 3, pp. 679-693, 1997.

[35] W. Rushi, Z. Daqi, and P. Li, "Character recognition algorithm based on multi-layer excitation function quantum neural network," Data Acquisition and Processing, vol. 4, pp. 401-406, 2007.

[36] X.-F. Niu and W.-P. Ma, "A novel quantum neural network based on multi-level activation function," Laser Physics Letters, vol. 18, no. 2, article 025201, 2021. 